\title{
Acute net stress of young adult zebrafish (Danio rerio) is not sufficient to increase anxiety-like behavior and whole-body cortisol
}

\author{
Amy Aponte ${ }^{1}$, Maureen L Petrunich-Rutherford ${ }^{\text {Corresp. } 1}$ \\ 1 Department of Psychology, Indiana University Northwest, Gary, IN, United States \\ Corresponding Author: Maureen L Petrunich-Rutherford \\ Email address: mlpetrun@iun.edu
}

In recent years, the zebrafish (Danio rerio) has become a popular model to study the mechanisms of physiological and behavioral effects of stress, due to the similarity in neural structures and biochemical pathways between zebrafish and mammals. Previous research in this vertebrate animal model has demonstrated an increase in whole-body cortisol resulting from an acute (30-second) net handling stress, but it remains unclear whether such a stressor will concomitantly increase anxiety-like behavior. In addition, as the previous study examined the effects of this acute stressor in adult zebrafish after a brief period of isolation, it is unclear whether this stressor would be effective in eliciting cortisol increases in younger aged subjects without isolation. In the current study, young adult zebrafish (approximately 90 days post-fertilization) were briefly exposed to a net handling stressor and were subsequently subjected to either the novel tank test or the light/dark preference test. The novel tank test was used to measure exploration and habituation in response to a novel environment, and the light/dark preference test was used to measure locomotor activity and scototaxis behavior. All subjects were sacrificed 15 minutes post-stressor and were analyzed for whole-body levels of cortisol. Contrary to expectations, there was no effect of acute net handling on cortisol levels. Similarly, acute net handling did not significantly induce anxiety-like behavior during the novel tank test or the light/dark preference test. Our findings demonstrate that there are possible developmental differences in response to an acute net handling stress, as we did not observe alterations in hormonal or behavioral measures of anxiety in young adult zebrafish. Alternatively, if zebrafish are not isolated before the stressor, they may be more resilient to a brief acute stressor. These results suggest the need for a different or more intense acute stressor in order further explore neuroendocrine mechanisms and anxietylike behavior at this developmental stage in the zebrafish animal model. 


\section{Acute net stress of young adult zebrafish (Danio rerio) is not sufficient to}

2 increase anxiety-like behavior and whole-body cortisol

3

4 Amy Aponte ${ }^{1}$ and Maureen L. Petrunich-Rutherford ${ }^{1}$

5

$6 \quad{ }^{1}$ Department of Psychology, Indiana University Northwest, Gary, Indiana, USA

7

8 Corresponding Author:

9 Maureen L. Petrunich-Rutherford

10 Email address: mlpetrun@iun.edu

11

12

13

14

15

16

17

18

19

20

21

22

23

24 


\section{Abstract}

In recent years, the zebrafish (Danio rerio) has become a popular model to study the

27 mechanisms of physiological and behavioral effects of stress, due to the similarity in neural

28 structures and biochemical pathways between zebrafish and mammals. Previous research in this vertebrate animal model has demonstrated an increase in whole-body cortisol resulting from an acute (30-second) net handling stress, but it remains unclear whether such a stressor will concomitantly increase anxiety-like behavior. In addition, as the previous study examined the effects of this acute stressor in adult zebrafish after a brief period of isolation, it is unclear whether this stressor would be effective in eliciting cortisol increases in younger aged subjects without isolation. In the current study, young adult zebrafish (approximately 90 days postfertilization) were briefly exposed to a net handling stressor and were subsequently subjected to either the novel tank test or the light/dark preference test. The novel tank test was used to measure exploration and habituation in response to a novel environment, and the light/dark preference test was used to measure locomotor activity and scototaxis behavior. All subjects were sacrificed 15 minutes post-stressor and were analyzed for whole-body levels of cortisol.

40 Contrary to expectations, there was no effect of acute net handling on cortisol levels. Similarly, 41 acute net handling did not significantly induce anxiety-like behavior during the novel tank test or 42 the light/dark preference test. Our findings demonstrate that there are possible developmental 43 differences in response to an acute net handling stress, as we did not observe alterations in 44 hormonal or behavioral measures of anxiety in young adult zebrafish. Alternatively, if zebrafish 45 are not isolated before the stressor, they may be more resilient to a brief acute stressor. These 46 results suggest the need for a different or more intense acute stressor in order further explore 
47 neuroendocrine mechanisms and anxiety-like behavior at this developmental stage in the

48 zebrafish animal model.

49

\section{Introduction}

Among psychiatric disorders, anxiety disorders are the most common in adults in the

52 United States, with a lifetime prevalence rate at an estimated 28.8\% (Kessler et al., 2005). In

53 addition to immediate and potentially harmful long-term health effects, anxiety disorders present

54 an annual medical cost of $\$ 42.3$ to $\$ 46.6$ billion dollars in the United States (DuPont et al.,

55 1996). In an attempt to address both individual health concerns and ease the economic burden,

56 research in anxiety has focused on investigating the neural and endocrine mechanisms associated

57 with anxiety to better understand the pathology and treatment options associated with stress-

58 related conditions.

59 Brain structures such as the hypothalamus, amygdala, and hippocampus are responsible

60 for controlling and mediating the effects of stress (McEwen, 2007). When confronted with a

61 threatening situation, an organism experiences an innate stress response, consisting of a series of

62 physiological and behavioral changes that serve as coping mechanisms to ultimately return the

63 organism to a homeostatic state (Gold, 2015). The autonomic nervous system and hypothalamic-

64 pituitary-adrenal (HPA) axis serve important roles in regulating stress responses. Alterations in

65 the structure and/or function of the hypothalamus and the HPA axis are associated with anxiety

66 and other stress-related conditions (Faravelli et al., 2012; Terlevic et al., 2013; Zorn et al., 2017).

67 Studying the effects of stress on physiology and behavior is, therefore, imperative to

68 understanding and treating anxiety disorders. 
Animal models are critical for studying the behavior, neural, and endocrine responses to

70 stress exposure. Among vertebrates, the zebrafish (Danio rerio) is increasingly becoming a

71 model organism in biomedical research. In particular, the zebrafish model is rapidly gaining

72 popularity in neuroscience, largely because it requires inexpensive, simple handling, and

73 breeding is rapid and richly produces offspring (Gerlai, 2010). Most importantly, examination of

74 central nervous system anatomy, genomic sequences, and biochemical pathways in zebrafish

75 have been found to have similar mammalian homologs and further supports the use of zebrafish

76 as a model organism for studying neural mechanism of behaviors (Guo, 2009; Holzschuh et al.,

77 2001). With regards to stress research, the nervous and endocrine systems regulating biological

78 and behavioral responses to stress are highly conserved in zebrafish (Stewart et al., 2012). For

79 example, the hypothalamic-pituitary-interrenal (HPI) axis of zebrafish is analogous to the HPA

80 axis of mammals (Nesan and Vijayan, 2013; Wendelaar Bonga, 1997). When zebrafish

81 encounter stress, similar to mammals, the hypothalamus of the animal releases corticotropin

82 releasing hormone $(\mathrm{CRH})$ to stimulate the release of adrenocorticotropic hormone $(\mathrm{ACTH})$ from

83 the anterior pituitary. ACTH stimulates the production and release of cortisol into the circulation

84 from the interrenal cells (for a review of the zebrafish HPI axis, see Alsop \& Vijayan, 2009a).

85 Thus, the ability to measure basal and stress-induced cortisol neuroendocrine responses mirrors

86 mammalian responses and is a major advantage of the zebrafish model.

87 In addition, zebrafish are not exempt from the physiological and behavioral adaptations

88 in response to variable stressors, and different testing paradigms have been used to investigate

89 behaviors characteristic of anxiety. Among these models, the novel tank test has been a powerful

90 tool in analyzing habituation and motor activity (Raymond et al., 2012; Wong et al., 2010). In

91 conditions where zebrafish have not been exposed to factors that may induce anxiety-like 
92 behavior, fish generally increase exploratory behavior and decrease freezing across the 6 minutes

93 of the testing period (Cachat et al., 2010). Certain swimming patterns characteristic of anxiety,

94 such as a decrease in time that the zebrafish spends in the top half of the tank, a decrease in the

95 number of times the zebrafish enters the top, and an increase in latency to enter the upper half

96 have been observed as a result of anxiogenic factors (Cachat et al., 2010; Wong et al., 2010).

97 However, a preference for the bottom can depend on the transparency of the tank (R. E. Blaser \&

98 Rosemberg, 2012). Anxiolytic agents, on the other hand, produce opposite effects in the novel

99 tank test, such as a decreased latency to enter the top and increased entries to top (Egan et al.,

100 2009).

Another complementary testing paradigm for anxiety-like behavior is the light/dark

102 preference test (Kysil et al., 2017), in which the zebrafish is placed in a tank that typically

103 consists of an equally divided light (or white) and dark side. The light/dark preference test is

104 used to demonstrate scototaxis, or the preference of a dark compartment over a light

105 compartment, as a model of anxiety (Araújo et al., 2012; Champagne et al., 2010). This test

106 examines the instinctive motivation of the zebrafish to spend significantly more time in the dark

107 side of the tank, as a means to protect itself from potential predators, over its innate behavior to

108 explore a new environment (Maximino et al., 2010). The zebrafish is initially placed in the light

109 compartment and allowed to freely explore the tank; during this time, the time spent in the light

110 side, midline crossings, and distance traveled in the light side can be measured. Generally,

111 zebrafish show an initial preference for the dark compartment when illumination above the tank

112 is kept constant (Facciol et al., 2019, 2017). Anxiolytic drugs tend to decrease the time that the

113 zebrafish spend in the dark compartment and increase exploratory behavior, while anxiogenic 
114 drugs increase time spent in the dark compartment and decrease exploratory behavior (Magno et 115 al., 2015; Steenbergen et al., 2011).

116 Characterization of biochemical markers associated with behavioral changes is essential

117 for elucidating possible neuroadaptations elicited by stress exposure. Investigations in this area

118 will allow for a more complete understanding of possible vulnerability factors for stress-related

119 conditions or provide potential targets for pharmacological treatment options. A previous report

120 detailed neuroendocrine and neurochemical levels in zebrafish following an acute (30-second)

121 net handling stressor and reported a time-dependent increase in levels of whole body cortisol

122 with no change in brain levels of serotonin or dopamine in adult zebrafish (Tran et al., 2014).

123 However, anxiety-like behavior was not measured in any behavioral test in response to the acute

124 net stress in the aforementioned study (Tran et al., 2014). Despite evidence that zebrafish

125 demonstrate anxiety-like behaviors in response to physical stress and chemical anxiogenic

126 agents, such as an acute net chase or exposure to conspecific alarm pheromone (Mezzomo et al., 127 2019; Mocelin et al., 2015), it is unclear whether an acute net stressor will elicit behavioral

128 modification in zebrafish as measured by the novel tank test or the light/dark preference test.

129 The aim of the current study is to determine whether an acute net stressor is sufficient to

130 increase anxiety-like behavior and whole-body cortisol in young adult zebrafish. Young adult

131 zebrafish (90 days post-fertilization) were subjected to a brief net handling stressor and

132 subsequently exposed to the novel test tank or the light/dark preference test. We expected an

133 observable increase in anxiety-like behaviors in both of the behavioral tests immediately after the

134 acute stressor exposure, as well as an increase in whole body-cortisol in the young adult

135 zebrafish 15 minutes after the acute stressor. 
137 Methods

138 Animals. Wild-type zebrafish (Danio rerio) were bred from a stock population originally 139 obtained from Carolina Biological Supply (Burlington, NC). After fertilization, embryos were 140 washed with system water and kept at room temperature in $250 \mathrm{ml}$ beakers in embryo medium 141 (50 embryos/100 ml medium) until hatching (Westerfield, 2000). Larval zebrafish (from

142 hatching until 14 days post-fertilization (dpf)) were maintained at a density of 50 larvae/200 ml 143 stagnant water at room temperature. Larval fish were fed twice daily with dried, commercially144 available larval fish food, and were subject to gentle water exchanges once daily. On 15 dpf, fish 145 were gently moved to the system, a two-shelf, stand-alone housing rack (Aquaneering, San 146 Diego, CA, USA) with a slow drip. The drip was slowly increased every few days to acclimate

147 the fish to a steady stream of water by $30 \mathrm{dpf}$. The juvenile fish were maintained in $1.8 \mathrm{~L}$ tanks at 148 a density of approximately 5-6 fish/L until approximately $90 \mathrm{dpf}$ (the day of testing). There were 149 no visual barriers between home tanks. The system was maintained on a 14:10 h light/dark cycle, 150 water temperature of $26 \pm 2^{\circ} \mathrm{C}$, and $\mathrm{pH} 7.4 \pm 0.2$. After $30 \mathrm{dpf}$, fish were fed once daily with 151 commercially-available flake food. On the day of the experiment, the individual tanks housing 152 the mixed-sex young adult fish (approximately $90 \mathrm{dpf}$ ) were removed from the system and 153 moved to the experimental room. The fish were allowed to acclimate to the experimental room

154 for one hour before testing. All experimental procedures were conducted between 9:00 a.m. and 155 1:00 p.m. local time. As fish are considered exempt species according to the U.S. Animal

156 Welfare Act, this work did not require oversight by the Institutional Animal Care and Use 157 Committee (IACUC) of the institution. However, all procedures involving the care and use of the 158 animals were conducted according to established recommendations (Harper \& Lawrence, 2016; 159 National Research Council, 2011; Westerfield, 2000). 
Acute net stressor. Randomly selected individual fish in the experimental condition $(\mathrm{n}=$

161

162

163

164

165

166

167

168

169

170

171

172

173

174

175

176

177

178

179

180

181

182

20) were netted out of the home tank and suspended in the net above the water for 30 seconds

(Tran et al., 2014; Tran and Gerlai, 2015). A separate control group $(n=20)$ was not exposed to the acute net stressor. Half of each treatment group were subsequently exposed to either the novel tank test (Experiment 1) or the light/dark preference test (Experiment 2). Each sample was assigned a number upon selection from the home tank; the corresponding treatments for each sample were not revealed until after the automated behavioral analysis and cortisol assays were conducted.

Experiment 1: Novel tank test. After the acute net stressor (for fish in the stressed condition, $\mathrm{n}=10$ ) or directly from home tank (for fish in the control condition, $\mathrm{n}=10$ ), fish were individually netted and placed into a trapezoidal novel tank the same size and dimensions as the home tank (approximately $7 \mathrm{~cm}$ x $33 \mathrm{~cm}$ x $15 \mathrm{~cm}$, Aquaneering part number ZT180T) for six minutes. The behavior of the fish were recorded and subsequently analyzed with BehaviorCloud motion-tracking software (https://www.behaviorcloud.com/, San Diego, CA). Number of entries to the top of tank, time spent in top ( $\mathrm{sec})$, distance traveled in the top (cm), time spent in bottom ( $\mathrm{sec})$, distance traveled in the bottom $(\mathrm{cm})$, and immobility duration ( $\mathrm{sec}$ ) were used as markers of anxiety behavior. A fish demonstrating anxiety-like behavior is less likely to explore the top, more likely to stay in the bottom zone of the novel tank, and will demonstrate more freezing behavior. Total distance traveled $(\mathrm{cm})$ and mean speed $(\mathrm{cm} / \mathrm{sec})$ were measured to ensure the acute stressor did not compromise activity levels (Cachat et al., 2010). One sample from each group was excluded from behavioral analyses due to incomplete video files.

Experiment 2: Light/dark preference test. After the acute net stressor (for fish in the stressed condition $n=10$ ) or directly from home tank (for fish in the control condition, $n=10$ ), 
183 fish were individually netted and placed into a rectangular tank (approximately $15 \mathrm{~cm} \mathrm{x} 30 \mathrm{~cm} \times$ $18420 \mathrm{~cm}$ ) with a water depth of $10 \mathrm{~cm}$ for fifteen minutes. The dark side of the tank (sides and 185 bottom) was covered with black plastic aquarium background and the other side was left 186 uncovered, as modified from previously published procedures (Magno et al., 2015; Maximino et 187 al., 2010). The behavior of the fish were recorded and subsequently analyzed with 188 BehaviorCloud motion-tracking software (https://www.behaviorcloud.com/, San Diego, CA). 189 Number of entries to the light zone, total time spent in light zone (min), and immobility duration 190 (sec) were used as markers of anxiety behavior. Total distance traveled in the light side of the 191 tank $(\mathrm{cm})$ and velocity $(\mathrm{cm} / \mathrm{sec})$ were measured to ensure the acute stressor did not compromise 192 activity levels. One sample from the control group was excluded from behavioral analyses due to 193 an incomplete video file.

194 Euthanasia. In order to measure stress-induced cortisol responses at the peak of the 195 response (Ramsay et al., 2009; Tran et al., 2014), fifteen minutes after introduction to the 196 behavioral test, each fish was placed individually in a $50 \mathrm{~mL}$ beaker containing $0.1 \%(100 \mathrm{mg} / \mathrm{L})$ 197 clove oil in system water. Death was determined upon visual examination for cessation of 198 opercular (gill) movement and non-response to tactile stimulation (Davis et al., 2015). The 199 whole-body samples were gently dried, then stored in individual microcentrifuge tubes at $-20^{\circ} \mathrm{C}$. 202 and experiment 2 were extracted and determined in independent procedures. Briefly, whole203 body samples were thawed and weighed, then homogenized in $1 \mathrm{ml}$ ice-cold $25 \mathrm{mM}$ PBS buffer. 204 Diethyl ether $(5 \mathrm{ml})$ was added to the homogenates to extract the cortisol. After centrifugation, 205 the organic layer containing the cortisol was transferred to a new test tube. The 
206 ether/centrifugation step was repeated twice; all ether layers from each sample were collected in 207 a single tube. The samples from Experiment 1 were allowed to dry at room temperature under a 208 fume hood until the volatile compounds evaporated; samples from Experiment 2 were dried with 209 a light stream of air under the fume hood. In both procedures, samples were dried until a yellow 210 oil containing cortisol remained.

After the evaporation, $1 \mathrm{ml} 25 \mathrm{mM}$ PBS was added to the lipid-containing extract in each 212 tube. To determine cortisol levels, a cortisol enzyme-linked immunosorbent assay (ELISA) was 213 used (Salimetrics, State College, PA) as per the manufacturer's instructions. Cortisol levels were 214 normalized to whole-body weight and are expressed as ng cortisol/g whole-body weight. For 215 Experiment 1, three samples (one from the control group and two from the stress group) were 216 removed from the cortisol analysis due to issues with the extraction procedure. In Experiment 2, 217 one sample from the control group was removed from the analysis due to an extraction error. 218 Statistics. The data are presented as group means and the standard errors of the mean 219 (SEM). Overall behavioral measures and cortisol levels were analyzed by t-tests for independent 220 means. The behavioral data was also analyzed as a function of time (one and three minute bins 221 for the novel tank test and the light/dark preference test, respectively) and analyzed with a 222 repeated-measures ANOVA with Greenhouse-Geisser sphericity correction if Mauchly's test of 223 sphericity indicated a violation of the sphericity assumption. JASP software (https://jasp224 stats.org/, Amsterdam, The Netherlands) was used for statistical analyses. A significance value 225 of $\mathrm{p}<0.05$ was used as the criterion for a result to reach statistical significance.

\section{Results}


228 Experiment 1: Behavioral measures in the novel tank test and whole-body cortisol levels were

229 not altered in response to acute net stress in young adult zebrafish.

230

Motor activity. Young adult zebrafish exposed to an acute net stressor did not show any

231 differences in the total distance traveled $(\mathrm{cm})$ or mean speed $(\mathrm{cm} / \mathrm{sec})$ in the novel tank test

232

compared to control fish (Table 1). A t-test for independent means indicated no significant effect

233 of acute stress for either the total distance traveled $(\mathrm{t}(16)=-0.169, \mathrm{p}=0.868)$ or mean speed

$234(\mathrm{t}(16)=1.497, \mathrm{p}=0.154)$. When the total distance data was broken down into six 60-second bins

235 (Figure 1A) and analyzed with a repeated-measures ANOVA, there was no effect of treatment

$236(\mathrm{~F}(1,16)=0.029, \mathrm{p}=0.868)$, no effect of time $(\mathrm{F}(2.284,36.546)=1.246, \mathrm{p}=0.303)$, and no

237 interaction between treatment and time $(F(2.284,36.546)=0.933, p=0.413)$. For mean speed

238 over the first six minutes after being introduced into the novel tank (Figure 1B), there was no

239 effect of treatment $(\mathrm{F}(1,16)=2.242, \mathrm{p}=0.154)$, a significant effect of time $(\mathrm{F}(3.327,53.225)=$

$2407.907, \mathrm{p}<0.001)$, and no interaction between treatment and time $(\mathrm{F}(3.327,53.225)=0.741, \mathrm{p}=$

$2410.545)$. Generally, the mean speed of the zebrafish decreased across the task, but there was no

242 effect of treatment.

Immobility (freezing). According to a t-test for independent means, young adult zebrafish

244

exposed to an acute net stressor did not show any overall differences in immobility across the six

245 minutes of the novel tank test $($ Table $1 ; \mathrm{t}(16)=1.056, \mathrm{p}=0.307)$. When immobility was

246 analyzed by minute in the novel tank test (Figure 2$)$, there was no effect of treatment $(\mathrm{F}(1,16)=$

$2471.115, \mathrm{p}=0.307)$, a significant effect of time $(\mathrm{F}(2.854,45.667)=4.998, \mathrm{p}=0.005)$, and no

248 interaction between treatment and time $(\mathrm{F}(2.854,45.667)=0.637, \mathrm{p}=0.588)$. The time that the

249 zebrafish spent immobile decreased across the task, but there was no effect of treatment on this

250 measure.

Peer] reviewing PDF | (2019:04:36634:1:0:NEW 2 Jul 2019) 
252 show any differences in time spent in the top zone ( $\mathrm{sec}$ ), the distance traveled in the top zone $253(\mathrm{~cm})$, or the number of entries to the top in the novel tank test compared to control fish (Table 1).

254 A t-test for independent means indicated no significant effect of condition for the time spent in 255 the top zone $(\mathrm{t}(16)=-0.158, \mathrm{p}=0.877)$, the distance traveled in the top zone $(\mathrm{t}(16)=-0.420, \mathrm{p}=$ $2560.680)$, or number of entries to the top $(\mathrm{t}(16)=-0.688, \mathrm{p}=0.501)$. When the time spent in the 257 top zone was broken down into six 60-second bins (Figure 3A) and analyzed with a repeated258 measures ANOVA, there was no effect of treatment $(F(1,16)=0.025, p=0.877)$, no effect of 259 time $(\mathrm{F}(2.586,41.382)=0.677, \mathrm{p}=0.550)$, and no interaction between treatment and time $260(\mathrm{~F}(2.586,41.382)=1.871, \mathrm{p}=0.156)$. For the distance traveled in the top zone (Figure 3B), 261 there was no effect of treatment $(F(1,16)=0.176, p=0.680)$, a significant effect of time 262 $(\mathrm{F}(2.522,40.358)=3.339, \mathrm{p}=0.035)$, and no interaction between treatment and time $(\mathrm{F}(2.522$, $40.358)=0.975, p=0.402$ ). For the number of entries to the top zone (Figure 3C), there was no effect of treatment $(F(1,16)=0.473, p=0.501)$, a significant effect of time $(F(5,80)=3.293, p=$ 0.009), and no interaction between treatment and time $(F(5,80)=0.399, \mathrm{p}=0.848)$. Generally, the fish explored the top zone of the novel tank less across the task, but there was no effect of treatment on upper zone exploration.

Young adult zebrafish exposed to an acute net stressor did not show any differences in 269 time spent in the bottom zone $(\mathrm{sec})$ and the distance traveled in the bottom zone $(\mathrm{cm})($ Table 1$)$.

270 A t-test for independent means indicated no significant effect of condition for the time spent in 271 the bottom zone $(\mathrm{t}(16)=0.161, \mathrm{p}=0.874)$ and the distance traveled in the bottom zone $(\mathrm{t}(16)=-$ $2720.035, \mathrm{p}=0.972)$. When the time spent in the bottom zone was broken down into six 60 -second 273 bins (Figure 4A) and analyzed with a repeated-measures ANOVA, there was no effect of 
274 treatment $(\mathrm{F}(1,16)=0.026, \mathrm{p}=0.874)$, no effect of time $(\mathrm{F}(2.589,41.417)=0.698, \mathrm{p}=0.538)$,

275 and no interaction between treatment and time $(F(2.589,41.417)=1.864, p=0.158)$. For the

276 distance traveled in the bottom zone (Figure 4B), there was no effect of treatment $(F(1,16)=$

277 0.001, $\mathrm{p}=0.972)$, no effect of time $(\mathrm{F}(2.519,40.310)=1.584, \mathrm{p}=0.213)$, and no interaction

278 between treatment and time $(\mathrm{F}(2.519,40.310)=1.919, \mathrm{p}=0.150)$.

279 Whole-body cortisol. Young adult zebrafish exposed to an acute net stressor and then

280 subsequently placed in the novel tank test did not show any differences in whole-body cortisol

281 levels compared to control fish (Figure 5). A t-test for independent means indicated no

282 significant effect of acute stress for cortisol levels $(\mathrm{t}(15)=-0.079, \mathrm{p}=0.938)$.

283 Experiment 2: Behavioral measures in the light/dark preference test and whole-body cortisol

284 levels were not altered by acute net stress in young adult zebrafish.

285

Motor activity. Young adult zebrafish exposed to an acute net stressor did not show any

286 differences in the overall total distance traveled $(\mathrm{cm})$ or mean speed $(\mathrm{cm} / \mathrm{sec})$ in the light/dark

287 preference test compared to control fish (Table 2). A t-test for independent means indicated no

288 significant effect of acute stress for either the total distance traveled $(\mathrm{t}(17)=-0.406, \mathrm{p}=0.689)$

289 or mean speed $(\mathrm{t}(17)=0.094, \mathrm{p}=0.926)$. When the total distance data was broken down into

290 five 3-minute bins (Figure 6A) and analyzed with a repeated-measures ANOVA, there was no

291 effect of treatment $(F(1,17)=0.165, p=0.689)$, a significant effect of time $(F(2.614,44.439, p<$

$2920.001)$, and a significant interaction between treatment and time $(\mathrm{F}(2.614,44.439)=2.943, \mathrm{p}=$

293 0.050). For mean speed (Figure 6B), there was no effect of treatment $(F(1,17)=0.007, p=$

294 0.935), a significant effect of time $(F(2.371,40.307)=6.747, p=0.002)$, and no interaction

295 between treatment and time $(\mathrm{F}(2.371,40.307)=2.039, \mathrm{p}=0.136)$. Generally, the overall motor 
296 activity of the zebrafish changed across the 15-minute task, but there was no effect of treatment 297 on the activity.

298 Immobility (freezing). Young adult zebrafish exposed to an acute net stressor did not 299 show any differences in the time spent immobile in the light/dark preference test compared to 300 control fish (Table 2). A t-test for independent means indicated no significant effect of acute 301 stress on immobility time $(\mathrm{t}(17)=-0.200, \mathrm{p}=0.844)$. When immobility was analyzed by 3 302 minute bins across the light/dark preference test (Figure 7), there was no effect of treatment $303(\mathrm{~F}(1,17)=0.040, \mathrm{p}=0.844)$, no effect of time $(\mathrm{F}(1.289,21.906)=2.097, \mathrm{p}=0.159)$, and no 304 interaction between treatment and time $(F(1.289,21.906)=0.090, p=0.828)$. The time that the 305 zebrafish spent immobile decreased across the task, but there was no effect of treatment on this 306 measure. Exploratory behavior. Young adult zebrafish exposed to an acute net stressor spent less 308 time in the light zone in the light/dark preference test and entered the light zone fewer times 309 compared to control fish (Table 2); however, these differences did not reach statistical 310 significance $(\mathrm{t}(17)=-0.471, \mathrm{p}=0.644$ and $\mathrm{t}(17)=-0.852, \mathrm{p}=0.406$, respectively). When the 311 time spent in the light zone was broken down into five 3-minute bins (Figure 8A) and analyzed 312 with a repeated-measures ANOVA, there was no effect of treatment $(F(1,17)=0.222, p=0.644)$, 313 a significant effect of time $(F(4,68)=2.750, p=0.035)$, but no interaction between treatment 314 and time $(\mathrm{F}(4,68)=0.078, \mathrm{p}=0.989)$. For the number of entries to the light zone (Figure 8B), 315 there was no effect of treatment $(F(1,17)=0.725, \mathrm{p}=0.406)$, no effect of time $(\mathrm{F}(2.224,37.816)$ $316=0.307, \mathrm{p}=0.760)$, and no interaction between treatment and time $(\mathrm{F}(2.224,37.816)=1.763, \mathrm{p}$ $317=0.182$ ). Generally, the fish explored the light zone of the light/dark tank less across the task, 318 but there was no significant effect of treatment on light zone exploration. 
320

321

322 323

\section{Conclusions}

325

326

327

328

329

330

331

332 preference test.

subsequently placed in the light/dark preference test did not show any differences in whole-body cortisol levels compared to control fish (Figure 9). A t-test for independent means indicated no significant effect of acute stress for cortisol levels $(\mathrm{t}(17)=0.320, \mathrm{p}=0.753)$.

The goal of the current study was to demonstrate, in young adult zebrafish, that an acute net handling stressor would reliably increase whole-body cortisol levels, as was demonstrated in previous studies (Tran et al., 2014; Tran and Gerlai, 2015). In addition, young adult zebrafish in the current study were immediately subjected to either the novel tank test or the light/dark preference test to assess the behavioral impact of the acute stressor. In contrast to previous research, the acute net stress was not sufficient to significantly alter whole-body cortisol levels 15 minutes after the acute stressor in young adult zebrafish. In support of this finding, there was no difference in anxiety-like behaviors as measured by the novel tank test and the light/dark

These findings may illustrate possible developmental differences in the stress response in zebrafish and may indicate that young adult zebrafish exhibit resistance to certain effects of mild stress. Studies in rodents demonstrate that the late adolescent period is marked by resiliency to certain behavioral effects of stress (Jankord et al., 2011). In humans, major changes in the neuroanatomy and sensitivity of certain brain structures, specifically in those structures that mediate responses to the external environment, occur during the adolescent period (Andersen, 2003). The lack of behavioral and neuroendocrine changes in response to stress in the current study potentially implies that, similar to other vertebrates, young zebrafish exhibit a degree of 
342 resiliency to certain stressors. Thus, a stronger stressor, such as confinement in a small tube

343 (Abreu et al., 2017), may be required in order to elicit neuroendocrine and behavioral responses, 344 at least in young zebrafish.

345 The lack of significant findings in the behavioral tests may be attributable to variations in 346 experimental procedures and husbandry protocols rather than an absence of anxiety-like behavior 347 elicited by acute stress in the young adult zebrafish. For example, in the initial published studies 348 investigating the acute net stressor (Tran et al., 2014; Tran and Gerlai, 2015), a week of isolation 349 was conducted prior to the acute stressor. Because we were interested in investigating the 350 practicality of eliciting stress responses of specifically this stressor in young adult fish, we did 351 not replicate the isolation part of the methods. However, the results of the current study suggest 352 the acute stressor was not sufficient to elicit stress responses when young adult zebrafish are 353 housed in groups; thus, the effects of this specific acute stressor may only be observed in 354 combination with previous social isolation procedures. Other methodological differences may 355 complicate the comparison of results between laboratories. For example, a recent study indicates that the frequency of feeding may impact the expression of anxiety-like behavior, with feeding once a day eliciting more anxiety-like behavior compared to feeding twice per day (Dametto et al., 2018). Thus, the results of the current study may have been affected by laboratory husbandry protocols. In addition, in the light/dark preference test, some labs use a central compartment in 360 the testing tank and a 3-minute acclimation period (Araújo et al., 2012), whereas in the current 361 study, a central compartment was not used and the zebrafish were initially netted into the light 362 side. Another study used a water depth of $3 \mathrm{~cm}$ in the light/dark preference test (Gebauer et al., 363 2011), whereas the current experiment utilized a light/dark tank with $10 \mathrm{~cm}$ of water. Some 364 studies leave the light compartment uncovered, while others cover it with an opaque or white 
365 366

367

368

369

370

371

372

373

374

375

376

377

378

379

380

381

382

383

384

385

386

material. Thus, these differences may mean that the light/dark test was not sensitive enough to capture anxiety-like behavior in the younger subjects.

Nevertheless, in the current study, the acute net stressor was tested in two separate experiments, using two behavioral tests, and neither the novel tank test nor the light/dark preference test produced observable behavioral modifications. In addition, the acute net stress did not elicit any observable changes in whole-body cortisol levels 15 minutes post-stressor in either of our experiments. It is entirely possible that young zebrafish have a different trajectory of whole-body cortisol release compared to adults (Abreu et al., 2014; Pavlidis et al., 2015; Ramsay et al., 2009; Tran et al., 2014), with the peak occurring before or after 15 minutes poststressor; however, the lack of effects observed in the behavioral tests strengthen the finding that the acute net stressor is not robust enough on its own to elicit stress responses in young adult zebrafish.

Overall, the current study provides additional information about the zebrafish as a model for studying physiological and behavioral effects of stress. Although many studies have investigated the effects brought upon by different stressors, literature in this field is typically limited to effects observed in either larval fish or during adulthood. Fewer studies have investigated stress regulation around the time of sexual maturation and in the young adult period (Alsop and Vijayan, 2009b; Baiamonte et al., 2016; Dipp et al., 2018; Forsatkar et al., 2017; Petrunich-Rutherford, 2019). Additional investigation of external factors and underlying mechanisms that mediate the effects of stress in the zebrafish can be used to further develop this animal as a practical model in neuroscience and further the current understandings of the plasticity and vulnerability of the stress response around the time of sexual maturation. 
389

390

391

392

393

394

395

396

397

398

399

400

401

402

403

404

405

406

407

408

409

410

411

412

413

414

415

416

417

418

419

420

421

\section{Conclusions}

This study was the first to determine that an acute net stressor is not sufficient to elicit anxiety-like behavioral responses in two different behavioral paradigms in young adult zebrafish.

Contrary to expectations, the acute net stressor did not elicit increases in whole-body levels of cortisol in young adult zebrafish. Thus, a different stressor or a more intense or prolonged stressor may be necessary to elicit anxiety-like behaviors and neuroendocrine responses in this age group. Furthermore, additional investigations in the time course of the stress-induced cortisol response of juvenile and young adult zebrafish are necessary to completely characterize stress responses in different developmental periods across the lifespan.

\section{References}

Abreu, M.S. de, Koakoski, G., Ferreira, D., Oliveira, T.A., Rosa, J.G.S. da, Gusso, D., Giacomini, A.C.V., Piato, A.L., Barcellos, L.J.G., 2014. Diazepam and fluoxetine decrease the stress response in zebrafish. PloS One 9, e103232. https://doi.org/10.1371/journal.pone.0103232

Abreu, M.S., Giacomini, A.C.V.V., Koakoski, G., Piato, A.L.S., Barcellos, L.J.G., 2017. Divergent effect of fluoxetine on the response to physical or chemical stressors in zebrafish. PeerJ 5, e3330. https://doi.org/10.7717/peerj.3330

Alsop, D., Vijayan, M., 2009a. The zebrafish stress axis: molecular fallout from the teleostspecific genome duplication event. Gen. Comp. Endocrinol. 161, 62-66. https://doi.org/10.1016/j.ygcen.2008.09.011

Alsop, D., Vijayan, M.M., 2009b. Molecular programming of the corticosteroid stress axis during zebrafish development. Comp. Biochem. Physiol. A. Mol. Integr. Physiol. 153, 49-54. https://doi.org/10.1016/j.cbpa.2008.12.008

Andersen, S.L., 2003. Trajectories of brain development: point of vulnerability or window of opportunity? Neurosci. Biobehav. Rev. 27, 3-18.

Araújo, J., Maximino, C., de Brito, T.M., da Silva, A.W.B., Oliveira, K.R.M., Batista, E. de J.O., Morato, S., Herculano, A.M., Gouveia, A., 2012. Behavioral and Pharmacological 
422

423

424

425

426

427

428

429

430

431

432

433

434

435

436

437

438

439

440

441

442

443

444

445

446

447

448

449

450

451

452

453

454

455

456

457

458

459

460

461

462

463

464

465

466

467
Aspects of Anxiety in the Light/Dark Preference Test, in: Zebrafish Protocols for Neurobehavioral Research, Neuromethods. Humana Press, Totowa NJ, pp. 191-202.

Baiamonte, M., Parker, M.O., Vinson, G.P., Brennan, C.H., 2016. Sustained Effects of

Developmental Exposure to Ethanol on Zebrafish Anxiety-Like Behaviour. PloS One 11, e0148425. https://doi.org/10.1371/journal.pone.0148425

Cachat, J., Stewart, A., Grossman, L., Gaikwad, S., Kadri, F., Chung, K.M., Wu, N., Wong, K., Roy, S., Suciu, C., Goodspeed, J., Elegante, M., Bartels, B., Elkhayat, S., Tien, D., Tan, J., Denmark, A., Gilder, T., Kyzar, E., Dileo, J., Frank, K., Chang, K., Utterback, E., Hart, P., Kalueff, A.V., 2010. Measuring behavioral and endocrine responses to novelty stress in adult zebrafish. Nat. Protoc. 5, 1786-1799.

https://doi.org/10.1038/nprot.2010.140

Canavello, P.R., Cachat, J.M., Beeson, E.C., Laffoon, A.L., Grimes, C., Haymore, W.A.M., Elegante, M.F., Bartels, B.K., Hart, P.C., Elkhayat, S.I., Tien, D.H., Mohnot, S., Amri, H., Kalueff, A.V., 2011. Measuring Endocrine (Cortisol) Responses of Zebrafish to Stress, in: Zebrafish Neurobehavioral Protocols, Neuromethods. pp. 135-142.

Champagne, D.L., Hoefnagels, C.C.M., de Kloet, R.E., Richardson, M.K., 2010. Translating rodent behavioral repertoire to zebrafish (Danio rerio): relevance for stress research. Behav. Brain Res. 214, 332-342. https://doi.org/10.1016/j.bbr.2010.06.001

Dametto, F.S., Fior, D., Idalencio, R., Rosa, J.G.S., Fagundes, M., Marqueze, A., Barreto, R.E., Piato, A., Barcellos, L.J.G., 2018. Feeding regimen modulates zebrafish behavior. PeerJ 6, e5343. https://doi.org/10.7717/peerj.5343

Davis, D.J., Klug, J., Hankins, M., Doerr, H.M., Monticelli, S.R., Song, A., Gillespie, C.H., Bryda, E.C., 2015. Effects of Clove Oil as a Euthanasia Agent on Blood Collection Efficiency and Serum Cortisol Levels in Danio rerio. J. Am. Assoc. Lab. Anim. Sci. JAALAS 54, 564-567.

Dipp, V.R., Valles, S., Ortiz-Kerbertt, H., Suarez, J.V., Bardullas, U., 2018. Neurobehavioral Alterations in Zebrafish Due to Long-Term Exposure to Low Doses of Inorganic Arsenic. Zebrafish 15, 575-585. https://doi.org/10.1089/zeb.2018.1627

DuPont, R.L., Rice, D.P., Miller, L.S., Shiraki, S.S., Rowland, C.R., Harwood, H.J., 1996. Economic costs of anxiety disorders. Anxiety 2, 167-172. https://doi.org/10.1002/(SICI)1522-7154(1996)2:4<167::AID-ANXI2>3.0.CO;2-L

Egan, R.J., Bergner, C.L., Hart, P.C., Cachat, J.M., Canavello, P.R., Elegante, M.F., Elkhayat, S.I., Bartels, B.K., Tien, A.K., Tien, D.H., Mohnot, S., Beeson, E., Glasgow, E., Amri, H., Zukowska, Z., Kalueff, A.V., 2009. Understanding behavioral and physiological phenotypes of stress and anxiety in zebrafish. Behav. Brain Res. 205, 38-44. https://doi.org/10.1016/j.bbr.2009.06.022 
468

469

470

471

472

473

474

475

476

477

478

479

480

481

482

483

484

485

486

487

488

489

490

491

492

493

494

495

496

497

498

499

500

501

502

503

504

505

506

507

508

509

510

511

512

513

Facciol, A., Iqbal, M., Eada, A., Tran, S., Gerlai, R., 2019. The light-dark task in zebrafish confuses two distinct factors: Interaction between background shade and illumination level preference. Pharmacol. Biochem. Behav. 179, 9-21.

https://doi.org/10.1016/j.pbb.2019.01.006

Facciol, A., Tran, S., Gerlai, R., 2017. Re-examining the factors affecting choice in the light-dark preference test in zebrafish. Behav. Brain Res. 327, 21-28.

https://doi.org/10.1016/j.bbr.2017.03.040

Faravelli, C., Lo Sauro, C., Lelli, L., Pietrini, F., Lazzeretti, L., Godini, L., Benni, L., Fioravanti, G., Talamba, G.A., Castellini, G., Ricca, V., 2012. The role of life events and HPA axis in anxiety disorders: a review. Curr. Pharm. Des. 18, 5663-5674.

Forsatkar, M.N., Nematollahi, M.A., Rafiee, G., Farahmand, H., Lawrence, C., 2017. Effects of the prebiotic mannan-oligosaccharide on the stress response of feed deprived zebrafish (Danio rerio). Physiol. Behav. 180, 70-77. https://doi.org/10.1016/j.physbeh.2017.08.010

Gebauer, D.L., Pagnussat, N., Piato, A.L., Schaefer, I.C., Bonan, C.D., Lara, D.R., 2011. Effects of anxiolytics in zebrafish: similarities and differences between benzodiazepines, buspirone and ethanol. Pharmacol. Biochem. Behav. 99, 480-486. https://doi.org/10.1016/j.pbb.2011.04.021

Gerlai, R., 2010. High-throughput behavioral screens: the first step towards finding genes involved in vertebrate brain function using zebrafish. Mol. Basel Switz. 15, 2609-2622. https://doi.org/10.3390/molecules15042609

Gold, P.W., 2015. The organization of the stress system and its dysregulation in depressive illness. Mol. Psychiatry 20, 32-47. https://doi.org/10.1038/mp.2014.163

Guo, S., 2009. Using zebrafish to assess the impact of drugs on neural development and function. Expert Opin. Drug Discov. 4, 715-726. https://doi.org/10.1517/17460440902988464

Holzschuh, J., Ryu, S., Aberger, F., Driever, W., 2001. Dopamine transporter expression distinguishes dopaminergic neurons from other catecholaminergic neurons in the developing zebrafish embryo. Mech. Dev. 101, 237-243.

Jankord, R., Solomon, M.B., Albertz, J., Flak, J.N., Zhang, R., Herman, J.P., 2011. Stress vulnerability during adolescent development in rats. Endocrinology 152, 629-638. https://doi.org/10.1210/en.2010-0658

Kessler, R.C., Berglund, P., Demler, O., Jin, R., Merikangas, K.R., Walters, E.E., 2005. Lifetime prevalence and age-of-onset distributions of DSM-IV disorders in the National Comorbidity Survey Replication. Arch. Gen. Psychiatry 62, 593-602. https://doi.org/10.1001/archpsyc.62.6.593

Kysil, E.V., Meshalkina, D.A., Frick, E.E., Echevarria, D.J., Rosemberg, D.B., Maximino, C.,

Peer] reviewing PDF | (2019:04:36634:1:0:NEW 2 Jul 2019) 
514

515

516

517

518

519

520

521

522

523

524

525

526

527

528

529

530

531

532

533

534

535

536

537

538

539

540

541

542

543

544

545

546

547

548

549

550

551

552

553

554

555

556

557

558

559

Lima, M.G., Abreu, M.S., Giacomini, A.C., Barcellos, L.J.G., Song, C., Kalueff, A.V., 2017. Comparative Analyses of Zebrafish Anxiety-Like Behavior Using Conflict-Based Novelty Tests. Zebrafish 14, 197-208. https://doi.org/10.1089/zeb.2016.1415

Magno, L.D.P., Fontes, A., Gonçalves, B.M.N., Gouveia, A., 2015. Pharmacological study of the light/dark preference test in zebrafish (Danio rerio): Waterborne administration. Pharmacol. Biochem. Behav. 135, 169-176. https://doi.org/10.1016/j.pbb.2015.05.014

Maximino, C., Marques de Brito, T., Dias, C.A.G. de M., Gouveia, A., Morato, S., 2010. Scototaxis as anxiety-like behavior in fish. Nat. Protoc. 5, 209-216. https://doi.org/10.1038/nprot.2009.225

McEwen, B.S., 2007. Physiology and neurobiology of stress and adaptation: central role of the brain. Physiol. Rev. 87, 873-904. https://doi.org/10.1152/physrev.00041.2006

Mezzomo, N.J., Fontana, B.D., Müller, T.E., Duarte, T., Quadros, V.A., Canzian, J., Pompermaier, A., Soares, S.M., Koakoski, G., Loro, V.L., Rosemberg, D.B., Barcellos, L.J.G., 2019. Taurine modulates the stress response in zebrafish. Horm. Behav. 109, 4452. https://doi.org/10.1016/j.yhbeh.2019.02.006

Mocelin, R., Herrmann, A.P., Marcon, M., Rambo, C.L., Rohden, A., Bevilaqua, F., de Abreu, M.S., Zanatta, L., Elisabetsky, E., Barcellos, L.J.G., Lara, D.R., Piato, A.L., 2015. Nacetylcysteine prevents stress-induced anxiety behavior in zebrafish. Pharmacol. Biochem. Behav. 139 Pt B, 121-126. https://doi.org/10.1016/j.pbb.2015.08.006

Nesan, D., Vijayan, M.M., 2013. Role of glucocorticoid in developmental programming: evidence from zebrafish. Gen. Comp. Endocrinol. 181, 35-44. https://doi.org/10.1016/j.ygcen.2012.10.006

Pavlidis, M., Theodoridi, A., Tsalafouta, A., 2015. Neuroendocrine regulation of the stress response in adult zebrafish, Danio rerio. Prog. Neuropsychopharmacol. Biol. Psychiatry 60, 121-131. https://doi.org/10.1016/j.pnpbp.2015.02.014

Petrunich-Rutherford, M.L., 2019. Chronic fluoxetine treatment of juvenile zebrafish (Danio rerio) does not elicit changes in basal cortisol levels and anxiety-like behavior in adulthood. PeerJ 7, e6407. https://doi.org/10.7717/peerj.6407

Ramsay, J.M., Feist, G.W., Varga, Z.M., Westerfield, M., Kent, M.L., Schreck, C.B., 2009. Whole-body cortisol response of zebrafish to acute net handling stress. Aquac. Amst. Neth. 297, 157-162.

Raymond, J., Chanin, S., Stewart, A.M., Kyzar, E., Gaikwad, S., Roth, A., Bruce, I., Fryar, C., Varga, D., Enriquez, J., Bagawandoss, S., Pham, M., Zapolsky, I., Green, J., Desmond, D., Hester, J., Kalueff, A.V., 2012. Assessing Habituation Phenotypes in Adult Zebrafish: Intra-and Inter-Trial Habituation in the Novel Tank Test, in: Zebrafish Protocols for Neurobehavioral Research, Neuromethods. Humana Press, Totowa NJ, pp. 273-285.

PeerJ reviewing PDF | (2019:04:36634:1:0:NEW 2 Jul 2019) 
560

561

562

563

564

565

566

567

568

569

570

571

572

573

574

575

576

577

578

579

580

581

582

583

584

585

586

587

588

589

590

591

592

593

594

595

596

597

598

599

600

601

602

603

604

605
Steenbergen, P.J., Richardson, M.K., Champagne, D.L., 2011. Patterns of avoidance behaviours in the light/dark preference test in young juvenile zebrafish: a pharmacological study. Behav. Brain Res. 222, 15-25. https://doi.org/10.1016/j.bbr.2011.03.025

Stewart, A., Gaikwad, S., Kyzar, E., Green, J., Roth, A., Kalueff, A.V., 2012. Modeling anxiety using adult zebrafish: a conceptual review. Neuropharmacology 62, 135-143. https://doi.org/10.1016/j.neuropharm.2011.07.037

Terlevic, R., Isola, M., Ragogna, M., Meduri, M., Canalaz, F., Perini, L., Rambaldelli, G., Travan, L., Crivellato, E., Tognin, S., Como, G., Zuiani, C., Bazzocchi, M., Balestrieri, M., Brambilla, P., 2013. Decreased hypothalamus volumes in generalized anxiety disorder but not in panic disorder. J. Affect. Disord. 146, 390-394. https://doi.org/10.1016/j.jad.2012.09.024

Tran, S., Chatterjee, D., Gerlai, R., 2014. Acute net stressor increases whole-body cortisol levels without altering whole-brain monoamines in zebrafish. Behav. Neurosci. 128, 621-624. https://doi.org/10.1037/bne0000005

Tran, S., Gerlai, R., 2015. Thirty-Second Net Stressor Task in Adult Zebrafish. Bio-Protoc. 5.

Wendelaar Bonga, S.E., 1997. The stress response in fish. Physiol. Rev. 77, 591-625. https://doi.org/10.1152/physrev.1997.77.3.591

Westerfield, M., 2000. The zebrafish book: A guide for the laboratory use of zebrafish (Danio rerio), 4th ed. University of Oregon Press, Eugene.

Wong, K., Elegante, M., Bartels, B., Elkhayat, S., Tien, D., Roy, S., Goodspeed, J., Suciu, C., Tan, J., Grimes, C., Chung, A., Rosenberg, M., Gaikwad, S., Denmark, A., Jackson, A., Kadri, F., Chung, K.M., Stewart, A., Gilder, T., Beeson, E., Zapolsky, I., Wu, N., Cachat, J., Kalueff, A.V., 2010. Analyzing habituation responses to novelty in zebrafish (Danio rerio). Behav. Brain Res. 208, 450-457. https://doi.org/10.1016/j.bbr.2009.12.023

Zorn, J.V., Schür, R.R., Boks, M.P., Kahn, R.S., Joëls, M., Vinkers, C.H., 2017. Cortisol stress reactivity across psychiatric disorders: A systematic review and meta-analysis. Psychoneuroendocrinology 77, 25-36. https://doi.org/10.1016/j.psyneuen.2016.11.036 
606

607

608

609

610

611

612

613

614

615

616

PeerJ reviewing PDF | (2019:04:36634:1:0:NEW 2 Jul 2019) 


\section{Table $\mathbf{1}$ (on next page)}

Behavioral measures of zebrafish in the novel tank test

Exposure to an acute net stressor did not significantly alter overall behavioral measures in the novel tank test (6 minutes) in young adult zebrafish compared to unstressed (control) fish. 
1 Table 1: Behavioral measures of zebrafish in the novel tank test

2 Exposure to an acute net stressor did not significantly alter overall behavioral measures in the

3 novel tank test (6 minutes) in young adult zebrafish compared to unstressed (control) fish.

4

\begin{tabular}{lccccccc}
\hline & \multicolumn{2}{c}{ Control } & Acute net stressor & & & \\
\multicolumn{1}{c}{ Variable } & M & SEM & M & SEM & t & df & p \\
& & & & & & & \\
\hline Total distance moved (cm) & 763.28 & 217.98 & 810.26 & 171.83 & -0.169 & 16 & 0.868 \\
Mean speed (cm/s) & 6.27 & 0.75 & 4.88 & 0.55 & 1.497 & 16 & 0.154 \\
Total time immobile (s) & 172.17 & 37.25 & 118.53 & 34.55 & 1.056 & 16 & 0.307 \\
Number of entries to top & 9.67 & 2.30 & 12.56 & 3.52 & -0.688 & 16 & 0.501 \\
Total time in top (s) & 61.11 & 16.19 & 65.78 & 24.79 & -0.158 & 16 & 0.877 \\
Distance in top (cm) & 119.33 & 50.02 & 157.54 & 75.96 & -0.420 & 16 & 0.680 \\
Total time in bottom (s) & 298.68 & 16.19 & 293.91 & 24.87 & 0.161 & 16 & 0.874 \\
Distance in bottom (cm) & 643.95 & 199.84 & 652.72 & 150.89 & -0.035 & 16 & 0.972 \\
\hline
\end{tabular}

5

6

7

8

9

10

11

12

13

14

15

16

17

18 
Figure 1

Measures of zebrafish motor activity in the novel tank test over time

Although the mean speed of young adult zebrafish generally decreased over time, acute net stress exposure did not alter (A) total distance traveled and (B) mean speed in the novel tank test. Bars indicate means of each group \pm SEM.
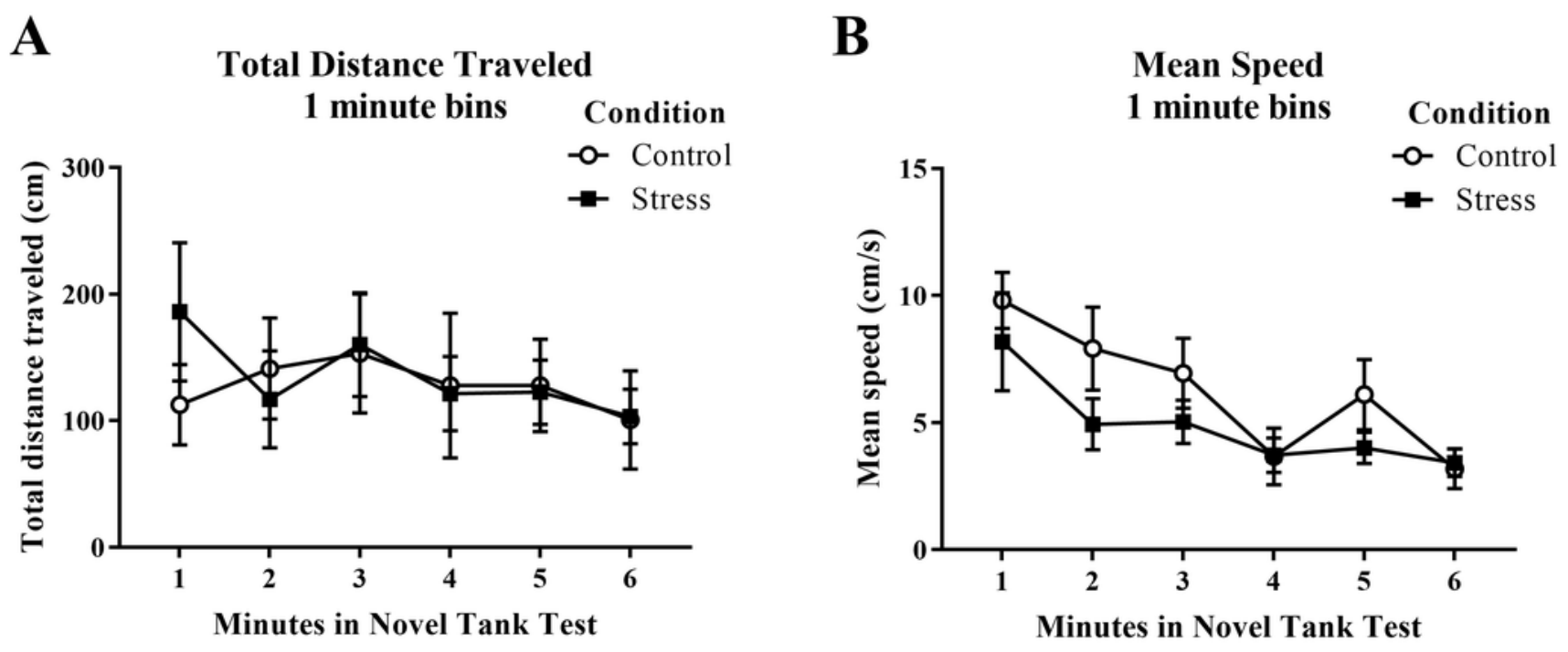
Figure 2

Measure of zebrafish freezing behavior in the novel tank test over time Although the time spent immobile decreased over time, acute net stress exposure did not alter the immobility of young adult zebrafish in the novel tank test. Bars indicate means of each group \pm SEM.

\section{Immobility \\ 1 minute bins}

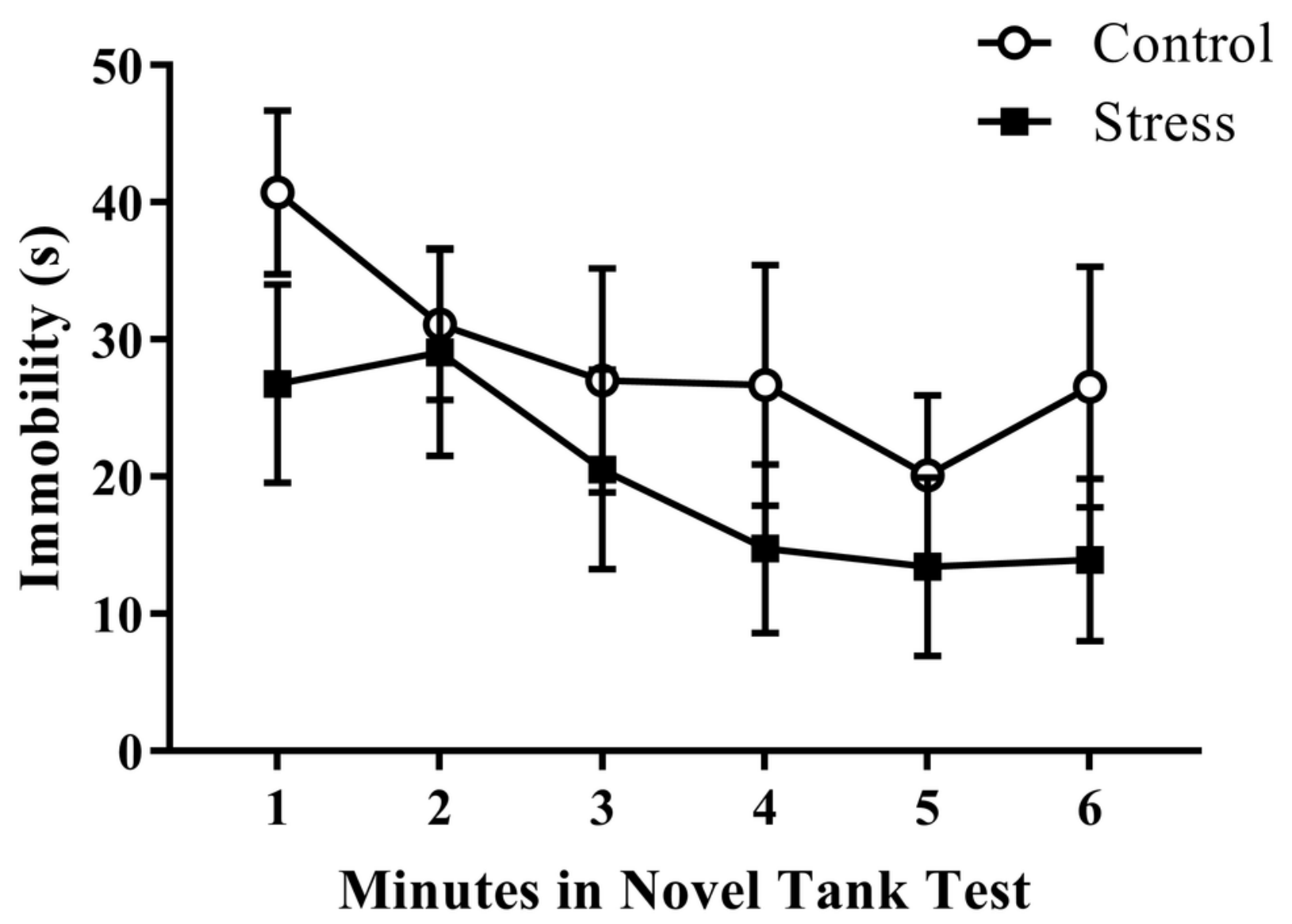


Figure 3

Measures of zebrafish activity in the top zone of the novel tank test over time Although young adult zebrafish generally explored the top zone less over the time of the task, acute net stress exposure did not alter (A) the time spent in the top zone, (B) the distance traveled in the top zone, and (C) the number of entries to the top zone of the novel tank test. Bars indicate means of each group \pm SEM.
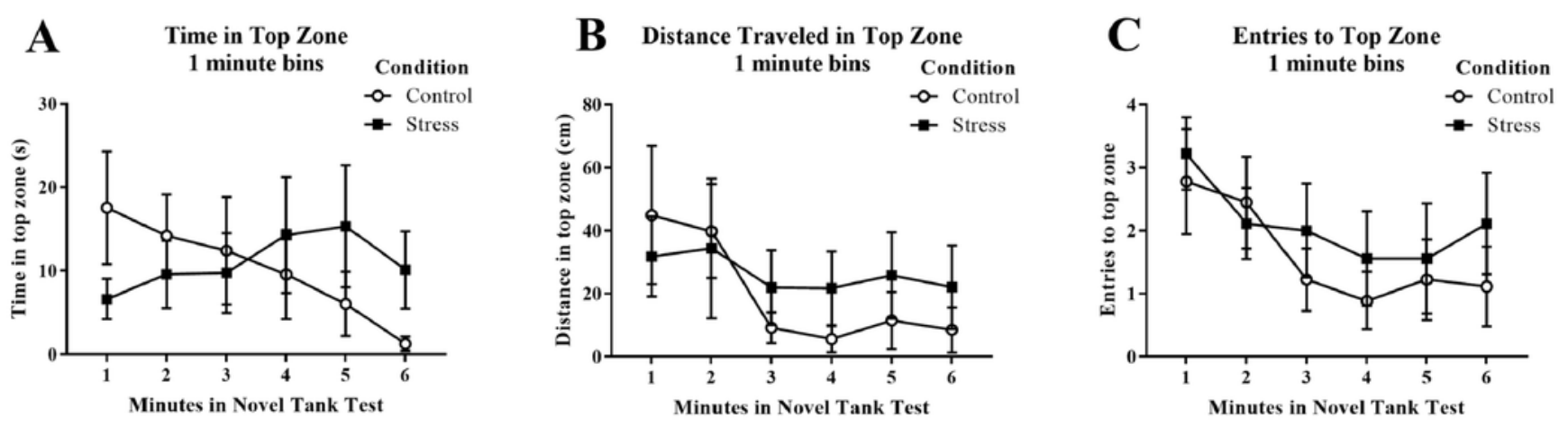
Figure 4

Measures of zebrafish activity in the bottom zone of the novel tank test over time Acute net stress exposure of young adult zebrafish did not alter $(A)$ the time spent in the bottom zone and (B) the distance traveled in the bottom zone of the novel tank test. Bars indicate means of each group \pm SEM.
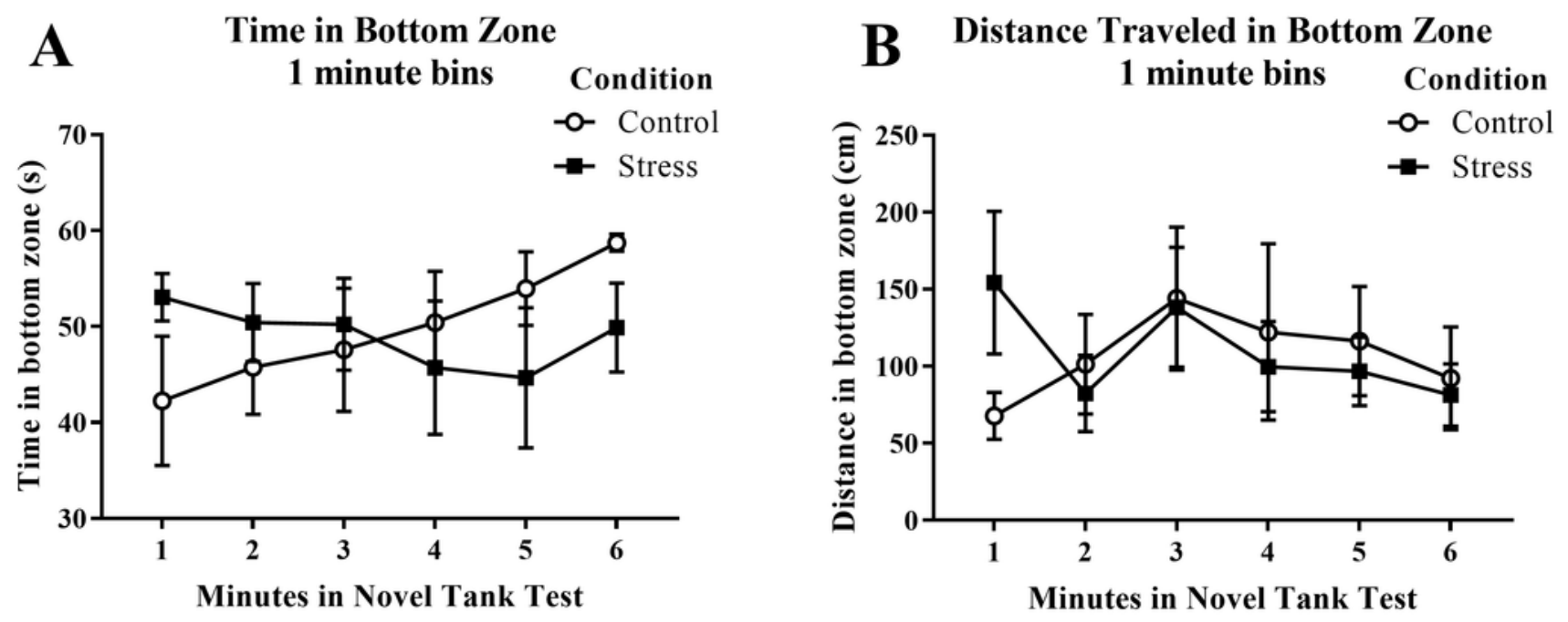


\section{Figure 5}

Measure of zebrafish neuroendocrine function after the novel tank test

Acute net stress exposure did not alter whole-body cortisol levels of young adult zebrafish in Experiment 1 (fish were sacrificed after the novel tank test). Bars indicate means of each group \pm SEM.

\section{Cortisol \\ (After Novel Tank Test)}

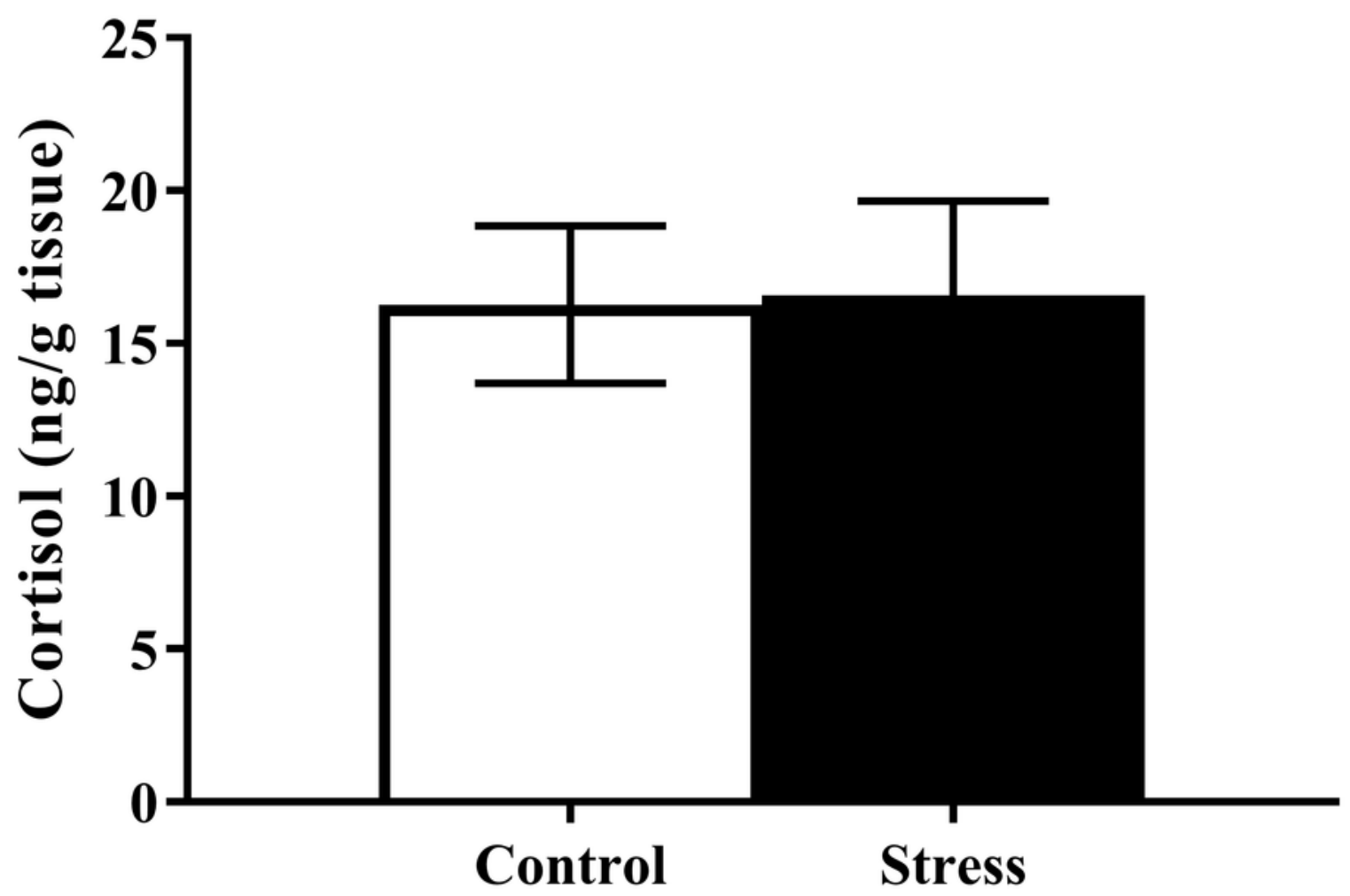

Treatment 


\section{Table 2 (on next page)}

Behavioral measures of zebrafish in the light/dark preference test

Exposure to an acute net stressor did not significantly alter overall behavioral measures in the light/dark preference test (15 minutes) in young adult zebrafish compared to unstressed (control) fish. 
1 Table 2: Behavioral measures of zebrafish in the light/dark preference test

2 Exposure to an acute net stressor did not significantly alter overall behavioral measures in the

3 light/dark preference test (15 minutes) in young adult zebrafish compared to unstressed (control)

4 fish.

5

\begin{tabular}{|c|c|c|c|c|c|c|c|}
\hline \multirow[b]{2}{*}{ Variable } & \multicolumn{2}{|c|}{ Control } & \multicolumn{2}{|c|}{ Acute net stressor } & \multirow[b]{2}{*}{$\mathrm{t}$} & \multirow[b]{2}{*}{$\mathrm{df}$} & \multirow[b]{2}{*}{$\mathrm{p}$} \\
\hline & M & SEM & M & SEM & & & \\
\hline Total distance moved (cm) & 2437.46 & 517.74 & 2131.06 & 542.21 & -0.406 & 17 & 0.689 \\
\hline Mean speed $(\mathrm{cm} / \mathrm{s})$ & 8.66 & 0.97 & 8.80 & 1.13 & 0.094 & 17 & 0.926 \\
\hline Total time immobile (s) & 48.06 & 24.29 & 42.69 & 13.09 & -0.200 & 17 & 0.844 \\
\hline Number of entries to light zone & 123.00 & 18.67 & 102.90 & 14.82 & -0.852 & 17 & 0.406 \\
\hline Total time in light zone (s) & 470.57 & 82.89 & 415.69 & 81.65 & -0.471 & 17 & 0.644 \\
\hline
\end{tabular}

6 
Figure 6

Measures of zebrafish motor activity in the light/dark preference test over time Although the total distance traveled and mean speed of young adult zebrafish changed across the task, acute net stress exposure did not alter (A) total distance traveled and (B) mean speed in the light/dark preference test. Bars indicate means of each group \pm SEM.
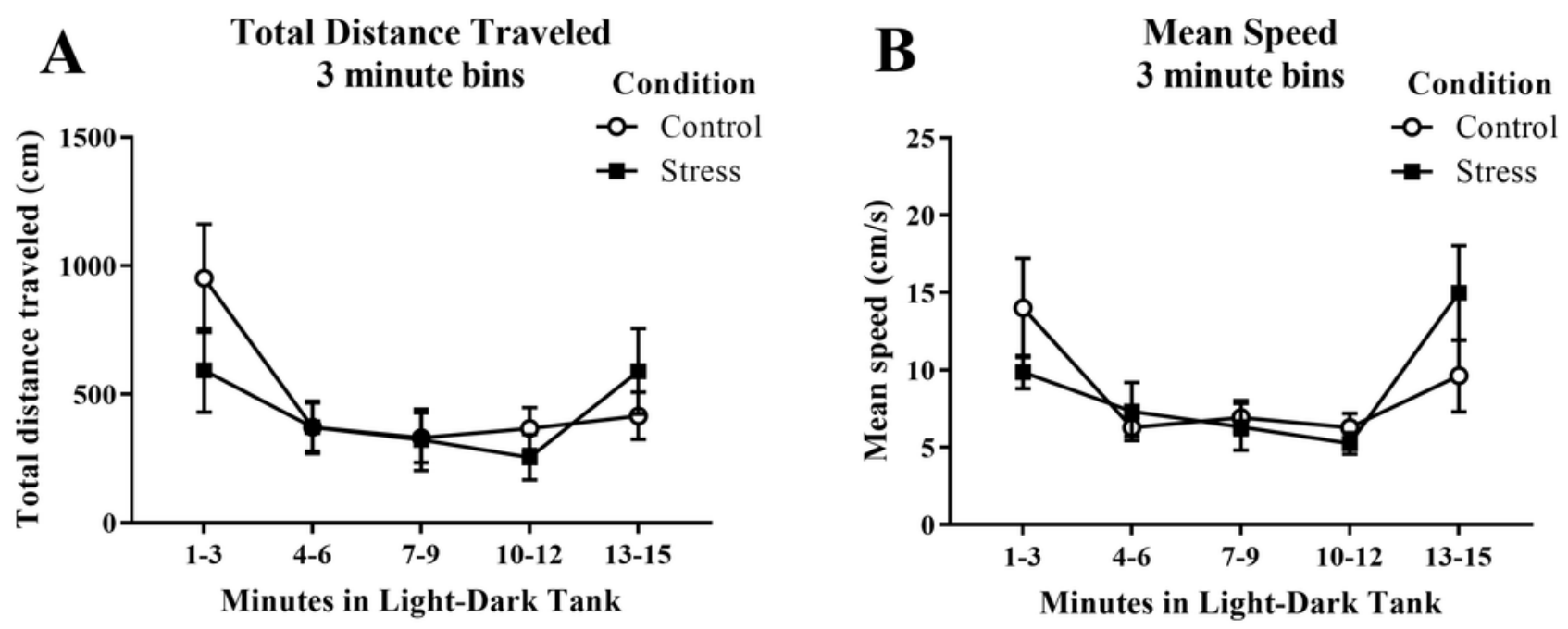
Figure 7

Measure of zebrafish freezing behavior in the light/dark preference test over time Acute net stress exposure did not alter the immobility of young adult zebrafish in the light/dark preference test. Bars indicate means of each group \pm SEM.

\section{Immobility \\ 3 minute bins}

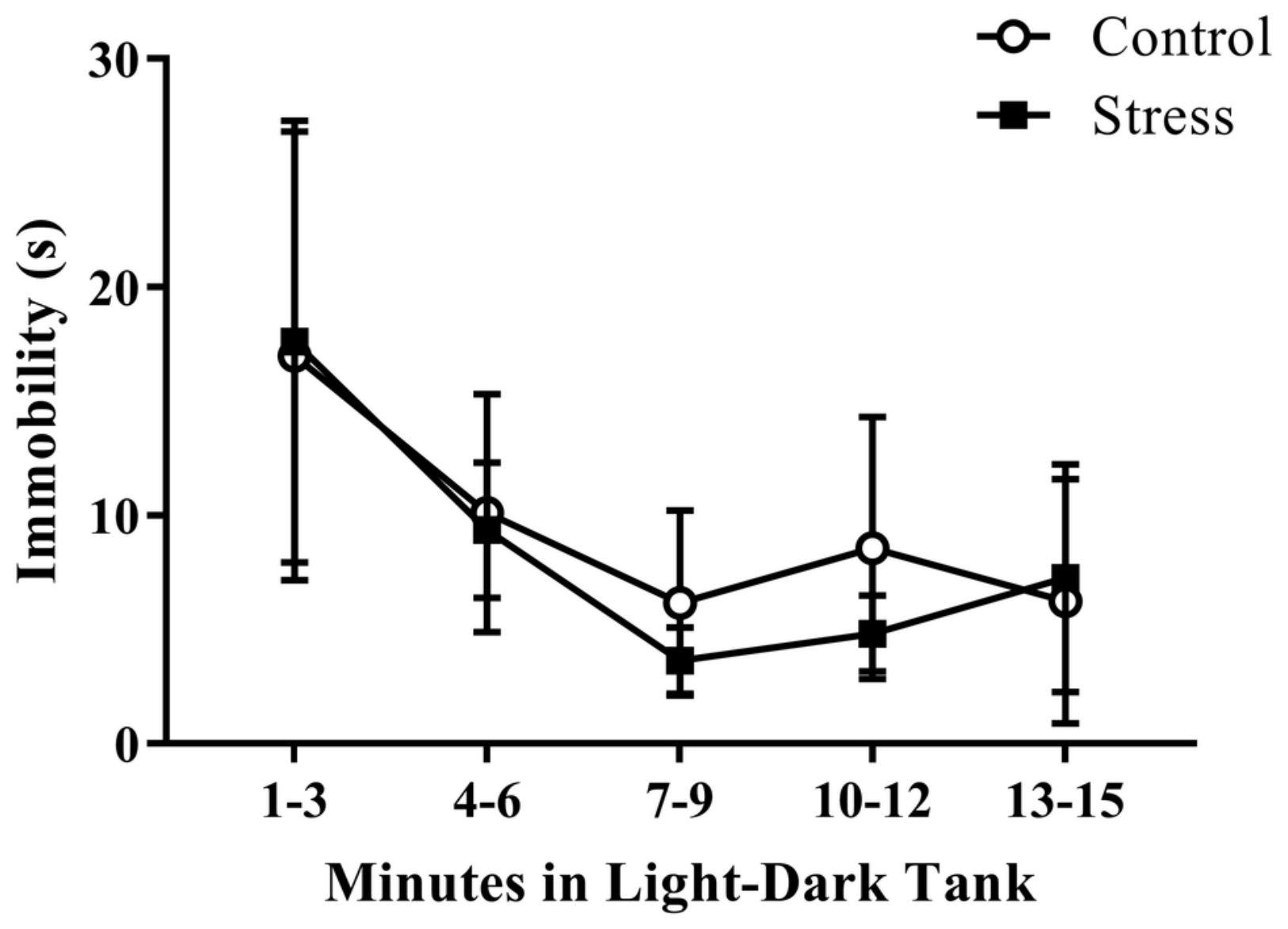


Figure 8

Measures of zebrafish activity in the light zone of the light/dark preference test over time

Although the amount of time the young adult zebrafish spent in the light zone generally decreased over time, acute net stress exposure did not alter $(A)$ the time spent in the light zone and (B) the distance traveled in the light zone of the light/dark preference test. Bars indicate means of each group \pm SEM.
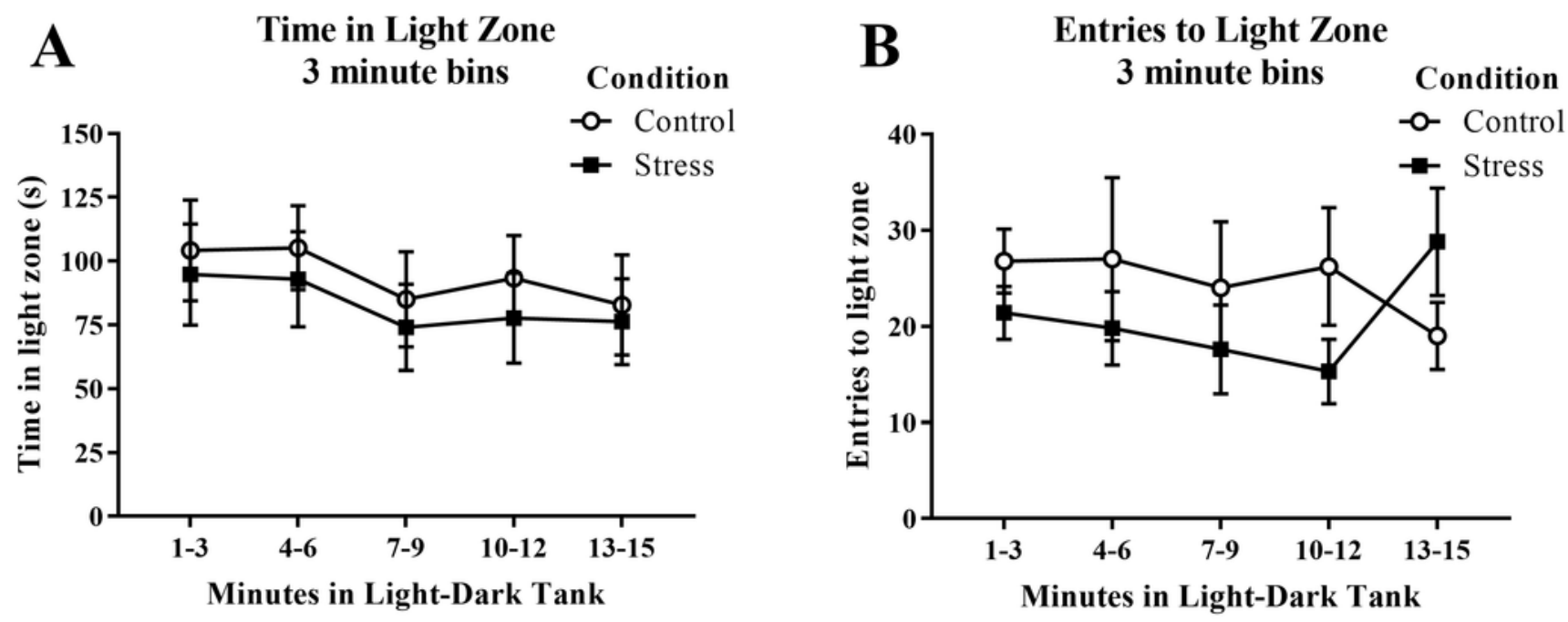


\section{Figure 9}

Measure of zebrafish neuroendocrine function after the light/dark preference test

Acute net stress exposure did not alter whole-body cortisol levels of young adult zebrafish in Experiment 2 (fish were sacrificed after the light/dark preference test). Bars indicate means of each group \pm SEM.

\section{Cortisol \\ (After Light-Dark Test)}

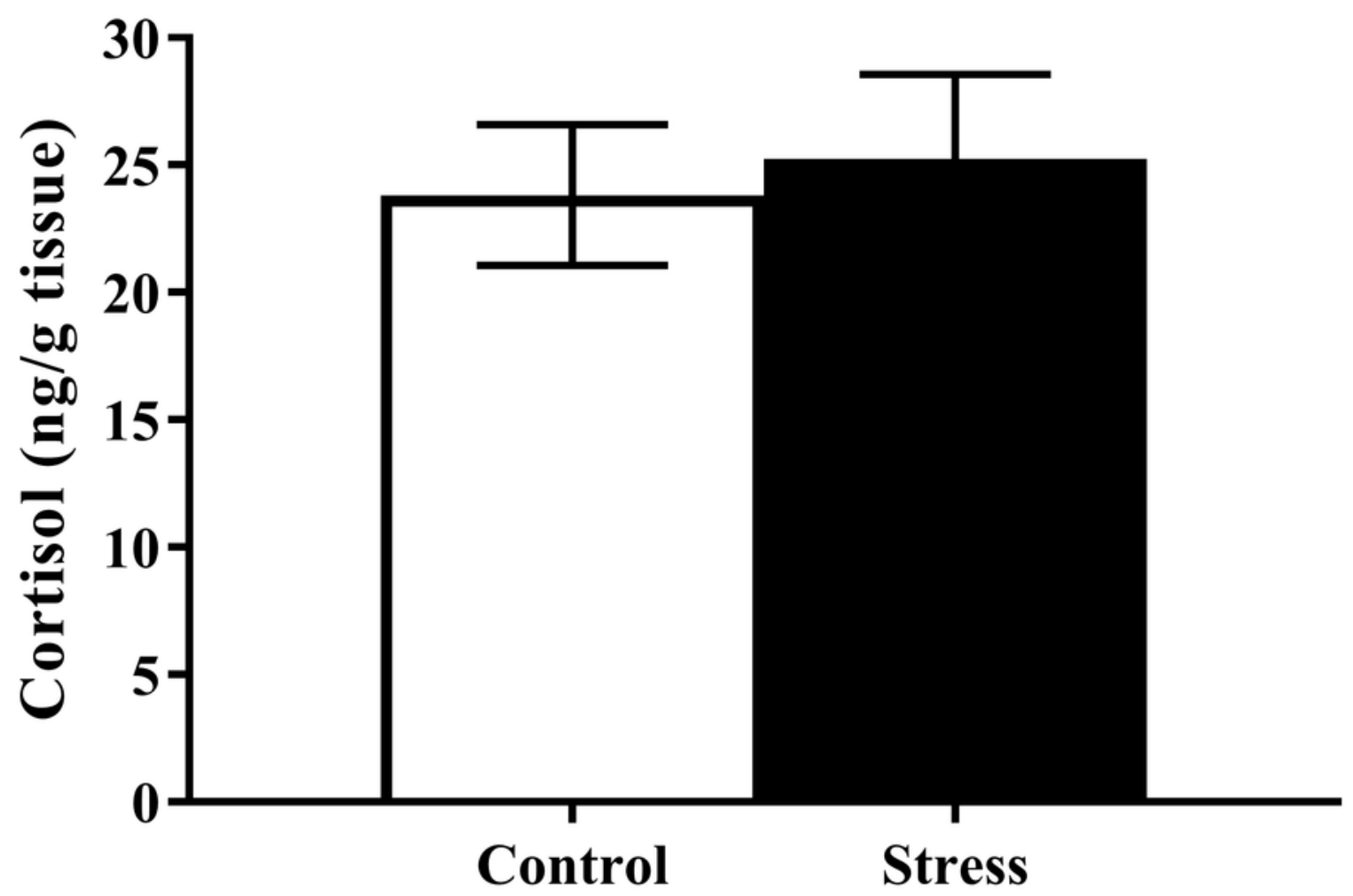

Treatment 